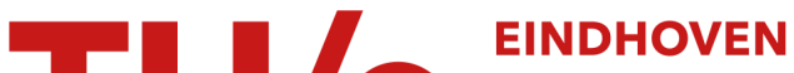 UNIVERSITY OF TECHNOLOGY
}

\section{Collapse interactions of finite-sized two-dimensional vortices}

\author{
Citation for published version (APA): \\ Vosbeek, P. W. C., van Geffen, J. H. G. M., Meleshko, V. V., \& Heijst, van, G. J. F. (1997). Collapse interactions \\ of finite-sized two-dimensional vortices. (RANA : reports on applied and numerical analysis; Vol. 9707). \\ Technische Universiteit Eindhoven.
}

\section{Document status and date:}

Published: 01/01/1997

\section{Document Version:}

Publisher's PDF, also known as Version of Record (includes final page, issue and volume numbers)

\section{Please check the document version of this publication:}

- A submitted manuscript is the version of the article upon submission and before peer-review. There can be important differences between the submitted version and the official published version of record. People interested in the research are advised to contact the author for the final version of the publication, or visit the $\mathrm{DOI}$ to the publisher's website.

- The final author version and the galley proof are versions of the publication after peer review.

- The final published version features the final layout of the paper including the volume, issue and page numbers.

Link to publication

\section{General rights}

Copyright and moral rights for the publications made accessible in the public portal are retained by the authors and/or other copyright owners and it is a condition of accessing publications that users recognise and abide by the legal requirements associated with these rights.

- Users may download and print one copy of any publication from the public portal for the purpose of private study or research.

- You may not further distribute the material or use it for any profit-making activity or commercial gain

- You may freely distribute the URL identifying the publication in the public portal.

If the publication is distributed under the terms of Article 25fa of the Dutch Copyright Act, indicated by the "Taverne" license above, please follow below link for the End User Agreement:

www.tue.nl/taverne

Take down policy

If you believe that this document breaches copyright please contact us at:

openaccess@tue.nl

providing details and we will investigate your claim. 
Collapse interactions of finite-sized two-dimensional vortices

by

P.W.C. Vosbeek, J.H.G.M. van Geffen

V.V. Meleshko and G.J.F. van Heijst

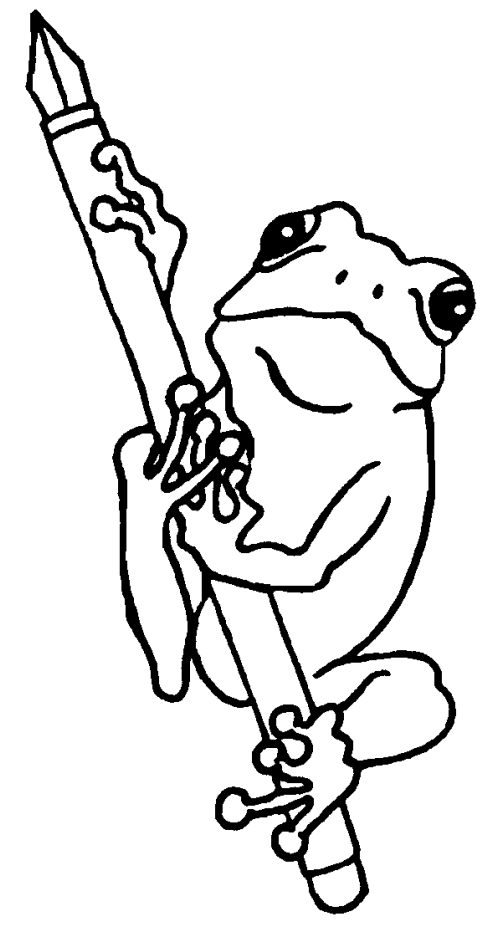


Reports on Applied and Numerical Analysis

Department of Mathematics and Computing Science

Eindhoven University of Technology

P.O. Box 513

5600 MB Eindhoven

The Netherlands

ISSN: 0926-4507 


\title{
Collapse interactions of finite-sized two-dimensional vortices
}

\author{
P.W.C. Vosbeek ${ }^{\mathrm{a})}$, J.H.G.M. van Geffen ${ }^{\mathrm{b}}$, \\ V.V. Meleshkoc) and G.J.F. van Heijst \\ Fluid Dynamics Laboratory \\ Department of Applied Physics \\ Eindhoven University of Technology \\ PO Box 513, 5600 MB Eindhoven, The Netherlands \\ tel. ++31402472673 , fax. ++31402464151 \\ E-mail: pauline@tnj.phys.tue.nl
}

\begin{abstract}
The point vortex model predicts that a certain configuration of three point vortices leads to a collapse of these vortices to one point. Numerical simulations have been performed to investigate the effect of a finite vortex size on this two-dimensional collapse interaction. The paper presents results obtained with contour dynamics simulations of patches of uniform vorticity, and results obtained with finite difference simulations of vortices with continuous properties. In addition, the effect of viscosity and the presence of impermeable domain boundaries are investigated. The results show that the motion of finite sized vortices is quite similar to the motion of point vortices as long as the mutual distance between the vortices is larger than their size. When the vortices are closer together their shapes start to deform and the subsequent evolution is different from that of the point vortices, and an actual collapse to one vortex does not take place.
\end{abstract}

Keywords: Vortex dynamics, Computational methods in fluid dynamics.

PACS numbers: 47.32.Cc, 47.11. $+\mathrm{j}$

\footnotetext{
a) also at Department of Mathematics and Computing Science, Eindhoven University of Technology.

b) present affiliation: Geophysical and Environmental Fluid Dynamics Laboratory, Department of Civil

Engineering, University of Dundee, Dundee DD1 4HN, UK.

c) also at the Institute of Hydrodynamics of the National Academy of Sciences, 252057 Kiev, Ukraine.
} 


\section{INTRODUCTION}

In his remarkable dissertation, Gröbli ${ }^{1}$ established the existence of a special self-similar motion of three point vortices of chosen intensity located initially at specific positions on an infinite plane (see also Aref et $a l^{2}{ }^{2}$ for historical and scientific background). In this special case the vortex trajectories have the form of logarithmic spirals with a common origin. Depending on the signs of the intensities of the vortices, they can either escape to infinity (as is the case for which Gröbli constructed a solution) or move inward and collapse in the origin in a finite time. Later studies Novikov and $\operatorname{Sedov}^{3}, \operatorname{Aref}^{4}, \operatorname{Kimura}^{5,6}$ ) have revealed more general conditions for point vortex collapse to occur, namely some specific relations between the intensities and the initial positions of the vortices. Although the collapse is in itself an interesting phenomenon, one may question the physical significance of this particular type of highly idealized point vortex interaction. The point vortex model has been proven to be very powerful in describing the interaction of (finite sized) vortices (see e.g. Meleshko and van Heijst ${ }^{7}$ ) and even the behaviour of dipolar and tripolar vortices in the presence of nonuniform background vorticity (see e.g. Velasco Fuentes et al. ${ }^{8,9}$ ). Yet, it is a priori not clear whether real vortices (with finite-sized, continuous vorticity distributions) show a collapse into a single vortex, as predicted by the point vortex model.

The present paper reports a numerical study of the effects of a finite vortex size on the collapse interaction. In the first set of simulations, the point vortices are replaced by initially circular vorticity patches (Rankine vortices) with corresponding circulation values. The evolution of these patches has been simulated using contour-dynamics, with the initial patch size (relative to the initial distances between the patch centres) as the main parameter of interest. In the second set of numerical simulations, the point vortices are replaced by vortices with a smooth vorticity distribution. The evolution of these vortices has been calculated by solving the two-dimensional vorticity equation (including the viscous terms) using a finite-difference method.

The main questions that will be addressed are: how are the trajectories and the shapes of the vortices affected by the finite size of the vortices? What is the influence of viscosity on the process of vortex interaction? Finally, what is the effect of solid domain boundaries 
on the vortex collapse for the case of point vortices?

The remainder of the paper is organized as follows. A brief description of the classical point-vortex collapse is given in Section II. The contour-dynamics simulations of the interaction of initially circular patches are discussed in Section III, while the results of the finite-difference simulations of real vortices with continuous vorticity distributions are presented in Section IV. Finally, some general conclusions are formulated in Section V.

\section{POINT VORTEX MOTION (ON AN INFINITE PLANE)}

The two-dimensional inviscid flow problem of $N$ interacting point vortices (strength $\gamma_{i}$, position $\left(x_{i}, y_{i}\right)$, with $\left.i=1, \ldots, N\right)$ in the unbounded $x, y$-plane consists of solving the nonlinear system of first-order differential equations

$$
\left\{\begin{array}{l}
u_{j}=\frac{d x_{j}}{d t}=-\frac{1}{2 \pi} \sum_{i=1}^{N} \frac{\gamma_{i}\left(y_{j}-y_{i}\right)}{r_{i j}^{2}}, \\
v_{j}=\frac{d y_{j}}{d t}=\frac{1}{2 \pi} \sum_{i=1}^{N} \frac{\gamma_{i}\left(x_{j}-x_{i}\right)}{r_{i j}^{2}},
\end{array}\right.
$$

where $r_{i j}^{2}=\left(x_{i}-x_{j}\right)^{2}+\left(y_{i}-y_{j}\right)^{2}$. The prime indicates omission of the singular term $i=j$ from the summation, and the initial configuration of the vortices is given by $x_{j}=x_{j}^{(0)}$, $y_{j}=y_{j}^{(0)}$ at $t=0$. It is well-known (see e.g. Batchelor ${ }^{10}$ ) that system (1) can be written in a Hamiltonian form with the Hamiltonian

$$
H=-\frac{1}{8 \pi} \sum_{i, j=1}^{N}{ }^{\prime} \gamma_{i} \gamma_{j} \log r_{i j}^{2}
$$

which is a conserved quantity. In addition to $H$, system (1) has three independent first integrals:

$$
Q=\sum_{i=1}^{N} \gamma_{i} x_{i}, \quad P=\sum_{i=1}^{N} \gamma_{i} y_{i}, \quad I=\sum_{i=1}^{N} \gamma_{i}\left(x_{i}^{2}+y_{i}^{2}\right) .
$$

A combination of the invariants (3) provides the invariant of motion

$$
L=\sum_{i, j=1}^{N} \gamma_{i} \gamma_{j} r_{i j}^{2}=2 \Gamma I-2\left(P^{2}+Q^{2}\right)
$$


with

$$
\Gamma=\sum_{i=1}^{N} \gamma_{i}
$$

the total circulation of the system. It was shown by Novikov and $\operatorname{Sedov}^{3}$, Aref ${ }^{4}$ and Kimura ${ }^{5}$ that if the two conditions

$$
L=0, \quad V=\sum_{i, j=1}^{N}{ }^{\prime} \gamma_{i} \gamma_{j}=0,
$$

are satisfied, a situation can exist where all distances between the vortices have the same time dependency,

$$
r_{i j}(t)=r_{i j}(0)(1+A t)^{\frac{1}{2}}
$$

with the constant $A$ depending on the initial conditions. If $A<0$, the vortices collide at time $T=-1 / A$.

We restrict our attention to the Gröbli case, with $N=3$ and

$$
\left\{\begin{array} { r l } 
{ x _ { 1 } ( 0 ) } & { = - 4 \alpha , } \\
{ y _ { 1 } ( 0 ) } & { = 0 , } \\
{ \gamma _ { 1 } } & { = - 3 \kappa , }
\end{array} \quad \left\{\begin{array} { r l } 
{ x _ { 2 } ( 0 ) } & { = - \frac { 9 \alpha } { 2 } , } \\
{ y _ { 2 } ( 0 ) } & { = \frac { 3 \alpha \sqrt { 3 } } { 2 } , } \\
{ \gamma _ { 2 } } & { = 2 \kappa , }
\end{array} \quad \left\{\begin{array}{r}
x_{3}(0)=\frac{\alpha}{2} \\
y_{3}(0)=\frac{\alpha \sqrt{3}}{2} \\
\gamma_{3}=-6 \kappa
\end{array}\right.\right.\right.
$$

where $\alpha$ and $\kappa$ are positive parameters. It is easy to check that the necessary conditions (6) are satisfied. The analytical solution of the equations of motion (1) satisfying these initial conditions is given by

$$
\begin{aligned}
& \left\{\begin{array}{l}
x_{1}(t)=4 \alpha(1-t / T)^{\frac{1}{2}} \cos (\pi+\vartheta(t)), \\
y_{1}(t)=4 \alpha(1-t / T)^{\frac{1}{2}} \sin (\pi+\vartheta(t)),
\end{array}\right. \\
& \left\{\begin{array}{l}
x_{2}(t)=3 \alpha \sqrt{3}(1-t / T)^{\frac{1}{2}} \cos \left(\frac{5}{6} \pi+\vartheta(t)\right), \\
y_{2}(t)=3 \alpha \sqrt{3}(1-t / T)^{\frac{1}{2}} \sin \left(\frac{5}{6} \pi+\vartheta(t)\right),
\end{array}\right. \\
& \left\{\begin{array}{l}
x_{3}(t)=\alpha(1-t / T)^{\frac{1}{2}} \cos \left(\frac{1}{3} \pi+\vartheta(t)\right), \\
y_{3}(t)=\alpha(1-t / T)^{\frac{1}{2}} \sin \left(\frac{1}{3} \pi+\vartheta(t)\right),
\end{array}\right.
\end{aligned}
$$


where $T$ and $\vartheta$ are given by

$$
\begin{aligned}
T & =\frac{14 \pi \alpha^{2}}{\kappa \sqrt{3}}, \\
\vartheta(t) & =\frac{5 \kappa}{2 \sqrt{3}} \log (1-t / T), \quad 0 \leqslant t \leqslant T .
\end{aligned}
$$

The trajectories of the point vortices are drawn in Figure 1 with solid lines, for the case where $\alpha=\kappa=1$ and thus $T=14 \pi / \sqrt{3}=25.3952$. In this figure, the three vortices at $t=0$ are connected with dashed lines to emphasize the shape of the triangle formed by them. Initially, this triangle has a right angle at vortex 1, and from (7) and (9) one can observe that this angle remains 90 degrees during the motion. In particular, the shape of the triangle does not change during the motion as a consequence of $(7)$.

In the next two sections computations with two different methods, viz. the contourdynamics method and the finite-difference method, are discussed. The point vortices are replaced by vortices with a finite size with initial strength and position according to (8) with $\alpha=\kappa=1$.

\section{COMPUTATIONS WITH CONTOUR-DYNAMICS}

Contour-dynamics (see Dritschel ${ }^{11,12}$, Zabusky et al. ${ }^{13}$ ) is based on the observation that the evolution of a patch of uniform vorticity in a two-dimensional flow of an incompressible, inviscid fluid is fully determined by the evolution of its boundary. The 2-D inviscid Euler equation written in terms of the stream function $(\psi)$ and the vorticity $(\omega)$ takes the following form:

$$
\frac{\partial \omega}{\partial t}+J(\omega, \psi)=0, \quad \nabla^{2} \psi=-\omega
$$

where $J$ is the Jacobian operator

$$
J(\omega, \psi)=\frac{\partial \psi}{\partial y} \frac{\partial \omega}{\partial x}-\frac{\partial \psi}{\partial x} \frac{\partial \omega}{\partial y},
$$

and $\nabla^{2}$ is the Laplace operator. The first equation expresses conservation of vorticity of a fluid particle. The solution of the second, the Poisson equation, in an infinite domain is 
formally given by

$$
\psi(\boldsymbol{x})=-\iint_{\mathbb{R}^{2}} \omega\left(\boldsymbol{x}^{\prime}\right) G\left(\boldsymbol{x} ; \boldsymbol{x}^{\prime}\right) d x^{\prime} d y^{\prime},
$$

where $G\left(\boldsymbol{x} ; \boldsymbol{x}^{\prime}\right)=\frac{1}{2 \pi} \ln \left\|\boldsymbol{x}-\boldsymbol{x}^{\prime}\right\|$, i.e. Green's function of the Laplace operator for an infinite domain, and $\boldsymbol{x}=(x, y)$.

For an initially piecewise uniform distribution of $\omega(\boldsymbol{x})$, it can be derived that the velocity field $\boldsymbol{u}(\boldsymbol{x})$, which is related to $\psi$ by

$$
u=\frac{\partial \psi}{\partial y}, \quad v=-\frac{\partial \psi}{\partial x},
$$

anywhere in the flow, and in particular on the contours $C_{m}$ where $\omega(\boldsymbol{x})$ is discontinuous, can be determined by the computation of a number contour integrals (see Dritschel ${ }^{11,12}$, Zabusky et $\left.a .^{13}\right)$ :

$$
\boldsymbol{u}(\boldsymbol{x})=-\sum_{m=1}^{M} \omega_{m} \oint_{C_{m}} G\left(\boldsymbol{x} ; \boldsymbol{x}^{\prime}\right) d \boldsymbol{x}^{\prime}
$$

Here, $\omega_{m}$ is the jump of vorticity when crossing the contour $C_{m}$ outward.

The contour integrals have to be computed numerically and the contours therefore have to be approximated by a finite, but adjustable, number of points. This technique is not discussed here; for details see Dritschel ${ }^{11,12}$, Vosbeek and Mattheij ${ }^{14}$. Integrating the velocities over a time step $\Delta t$ yields the positions of the boundaries after time step $\Delta t$ and thus the evolution of the regions of uniform vorticity can be calculated. The time integration is performed using a second order, symplectic, mid-point rule (see Sanz-Serna and $\mathrm{Calvo}^{15}$ ). The reason for choosing this scheme is that it conserves the area of the regions of uniform vorticity better than ordinary integration methods (see Vosbeek and Mattheij ${ }^{14}$ ).

\section{Results}

In order to study the effect of a finite vortex size on the three-vortex interaction in an infinite domain, contour-dynamics simulations were carried out with initially equal-sized circular patches (Rankine vortices) of radius $R$, at initial locations defined by (8), with 
$\alpha=\kappa=1$ and vorticity $\omega_{i}$ chosen in such a way that $\omega_{i} \pi R^{2}=\gamma_{i}$. In the first run, the vortex size was taken $R=0.50$. The trajectories of the centres of the three vorticity patches are drawn in Fig. 2 with solid lines; they were determined by computing for each patch at each time step the centre of vorticity by using a contour integral represention. Also drawn in this figure, but with dashed lines, are the trajectories of the corresponding point vortices initially located at the same positions. It is clear from this figure, that the trajectories of the vortices are in good agreement with the trajectories of the point vortices until $t=20$. After $t=20$, however, the trajectories deviate from those of the point vortices, and the angle at vortex 1 starts to increase. Close inspection of the evolving vorticity patches has revealed the deviation from the point-vortex trajectories to become noticeable approximately when the mutual distances between the patches becomes comparable to the patch sizes. Subsequently, the shape of some of the patches changes considerably. This is shown clearly by Fig. 3, where the boundaries of the vortices are drawn with solid black lines for six moments of time from $t=20$ on. Before $t=20$ (not shown) vortex 1 and vortex 2 become slightly elliptic (their aspect ratios remain much smaller than three), but this almost has no influence on their interaction behaviour. After $t=20$, however, vortex 1 (which is not the weakest) deforms very rapidly and is even completely torn apart at $t=25$. This deformation takes place very quickly, and can be explained by looking at the evolution of the strain rate of the velocity field.

According to Weiss ${ }^{16}$ and McWilliams ${ }^{17}$ the strain rate $Q$ is given by

$$
Q=\operatorname{tr}\left((\nabla \boldsymbol{u})^{2}\right)+\frac{1}{2} \omega^{2}
$$

where $\nabla \boldsymbol{u}$ is the stress tensor and $\operatorname{tr}$ is the trace. With the incompressibility condition $\nabla \cdot \boldsymbol{u}=0$, it easily follows that $\operatorname{tr}\left((\nabla \boldsymbol{u})^{2}\right)=-2 \operatorname{det}(\nabla \boldsymbol{u})$ so that

$$
Q=-2 \operatorname{det}(\nabla \boldsymbol{u})+\frac{1}{2} \omega^{2}
$$

Figure 3 shows, in addition to the boundaries of the vortex patches, also contourplots of this quantity $Q$; the dark grey regions are regions with strong strain, the white regions have (almost) no strain. 
The first feature that attracts attention, is the strong strain just outside vortex 3 , the strongest vortex. At $t=20$, this vortex is still almost circular, and the strain can thus be described with the analytical expression for $Q$ for a (circular) Rankine vortex

$$
Q= \begin{cases}0, & r<R, \\ \frac{1}{2} \omega^{2} \frac{R^{4}}{r^{4}}, & r>R\end{cases}
$$

where $R$ is the radius, $\omega$ the vorticity and $r$ the radial distance to the centre of the Rankine vortex. Just outside the vortex, the strain is strongest and equal to $\frac{1}{2} \omega^{2}$, and it decreases with $1 / r^{4}$.

The second feature that attracts attention, is the presence of a rather strong strain at the long ends of the more or less elliptical vortices 1 and 2 at $t=20$ and later. This feature can be described by the analytical expression for $Q$ for an (elliptic) Kirchhoff vortex, which can be derived from the expression of the stream function given by Lamb ${ }^{18}$. After some calculation, it follows that $Q$ is given by

$$
Q= \begin{cases}\frac{1}{2} \omega^{2} \frac{(a-b)^{2}}{(a+b)^{2}}, & \frac{x^{2}}{a^{2}}+\frac{y^{2}}{b^{2}}<1, \\ \frac{(2 a b \omega)^{2}}{\left(a^{2}-b^{2}\right)^{2}} \frac{\mathrm{e}^{-2 \xi}}{(\cosh (2 \xi)-\cos (2 \eta))}, & \frac{x^{2}}{a^{2}}+\frac{y^{2}}{b^{2}}>1,\end{cases}
$$

where $a$ and $b$ are the semi-major and semi-minor axis lengths of the ellipse, and $\xi$ and $\eta$ are elliptic coordinates which are related to the cartesian coordinates $x$ and $y$ by

$$
\begin{aligned}
& x=\left(a^{2}-b^{2}\right)^{\frac{1}{2}} \cosh (\xi) \cos (\eta), \\
& y=\left(a^{2}-b^{2}\right)^{\frac{1}{2}} \sinh (\xi) \sin (\eta) .
\end{aligned}
$$

From the expression for $Q$ outside the vortex it is clear that the strain is largest for $\eta=0$ and $\eta=\pi$ just outside the elliptical patch, i.e. at the long ends of the ellipse and smallest for $\eta=\pi / 2$ and $\eta=3 \pi / 2$. Note that inside the Kirchhoff vortex the strain is uniform and non-zero (unlike the Rankine vortex), its magnitude depending on the aspect ratio of the vortex. In particular, for aspect ratios smaller than 3 , the strain rate inside the vortex is smaller than the minimum value of it just outside the vortex, while for aspect ratios larger than 3 the strain rate inside the vortex is larger than the minimum value 
just outside it. To make this more clear, the value of $Q$ is plotted in a contour plot in Fig. 4, both for a vortex with aspect ratio 2 (Fig. 4(a)) and for a vortex with aspect ratio 4 (Fig. 4(b)). As in Fig. 3, dark grey regions are regions with large values of $Q$. Since a Kirchhoff vortex is not stable for aspect ratios larger than $3\left(\right.$ see Love $^{19}$, Dritschel ${ }^{20}$ ), this suggests that the internal strain can play a role in the deformation of the vortex. This leads to the third and probably most important feature that can be observed from Fig. 3: the presence of strain inside vortex 1 at $t=20$, which increases rapidly when time evolves. The increasing strain results in a strong deformation of vortex 1 while it is gradually wrapped around vortex 3 . Note that during this process also vortex 2 experiences substantial strain while it is becoming more elliptical. At $t=25$ this internal strain has disappeared almost completely and the patch is less elongated.

From the analytical expression (19) for the Kirchhoff vortex, it follows that the internal strain is partially due to the elongated vortex shape but the strain induced by the neighbouring vortices (external strain), which become quite close to vortex 1 from $t=20 \mathrm{on}$, will of course also contribute significantly. An approximation of this external strain can be obtained by using the elliptical vortex patch model described by Dritschel and de la Torre Juárez ${ }^{21}$. In this model vortices are represented by elliptical patches of uniform vorticity and they are forced to remain elliptical during the evolution. In Fig. 5 this model has been used for the same collapse configuration as in Fig. 3 (i.e. the same initially circular patches of uniform vorticity at the same positions). The solid lines represent the edges of the (elliptical) vortices; the cross in the centre of the vortices displays both the magnitude and the orientation of the external strain with extension along the solid axis and compression along the short-dashed axis. The strong externally induced strain in vortex 1 from $t=20$ up to $t=23$ causes the elongation of the vortex. Note that comparison of the contour dynamics simulations of Fig. 3 with this elliptical model shows hardly any difference until $t=21$. From this moment on, however, the trajectories only slightly deviate from those of the contour dynamics simulations while the deformations of the vortices show larger diferences.

It is obvious from Fig. 3 that a "collapse" of the patches into one single vortex patch 
does not take place: after $t=25$ the two surviving vortices move along a curved path as an asymmetric dipolar structure, carrying the dynamically insignificant filament of the original vortex 1 with it.

The evolution of the vortices has also been computed for initial patch radii $R=0.25$ and 0.75. Figure 6 shows the last part of the trajectories for $R=0.25$ (a) and $R=0.75$ (b). For the first case the trajectories agree very well with the trajectories of the point vortices until $t=24$, while for the latter the trajectories are seen to deviate already at $t=15$. This confirms the observation that the vortices no longer behave as point vortices once their mutual distance becomes of the order of their size $(2 R)$. The different initial radii apparently only influence the time scale: the deformations of the vortices are in all cases very similar, but start at different moments in time.

In the next section we discuss the vortex interaction for the case of finite-size vortices with smooth, continuous vorticity distributions. As an approximation to such realistic vortices, the continuous vorticity distribution was modelled with the contour-dynamics method by a nested set of initially circular constant-vorticity patches, with the maximum vorticity at the centre. In the additional simulations the number of vorticity levels was varied in the range 2 to 8 ; the vorticity levels and the patch sizes were chosen in such a way that the total circulation corresponds to that of the corresponding point vortex (see (8)). Although the shape deformations of the nested patches are slightly different from those of the single-level patches of the same radii in Fig. 3, the centroid trajectories were found to be very similar.

\section{COMPUTATIONS WITH A FINITE-DIFFERENCE METHOD}

The interaction behaviour of finite-sized vortices with a smooth vorticity distribution has been simulated numerically by using a finite-difference method to solve the 2-D viscous vorticity equation

$$
\frac{\partial \omega}{\partial t}+J(\omega, \psi)=\nu \nabla^{2} \omega, \quad \nabla^{2} \psi=-\omega
$$


with $\nu$ the kinematic viscosity. System (21) describes the evolution of an initial vorticity distribution $\omega(\boldsymbol{x}, t=0)$ subject to nonlinear and viscous effects. After making (21) dimensionless with a typical length scale $L_{0}$ and a typical time scale $T_{0}$, the familiar Reynolds number Re appears:

$$
R e=\frac{L_{0}^{2} / T_{0}}{\nu}=\frac{\Gamma_{0}}{\nu},
$$

where $\Gamma_{0}$ is a typical circulation of the vorticity distribution. In the following all typical scales are set equal to 1 , so that the Reynolds number in effect is $R e=1 / \nu$, and all quantities are given in dimensionless units.

The finite-difference method used here is based on a code by Orlandi and Verzicco Orlandi ${ }^{22}$, Verzicco et al. ${ }^{23}$ ) and applies a discretization of (21) on a rectangular grid in a finite rectangular domain. The time evolution is computed with an explicit third-order Runge-Kutta scheme, the viscous term is discretized with a Crank-Nicolson scheme and the nonlinear term by the Arakawa scheme. The Poisson equation in (21) is solved with a Fast Fourier And Cyclic Reduction routine.

The distributed monopolar vortices were initialized with monopoles of the so-called Bessel type:

$$
\omega= \begin{cases}\frac{(k R) \Gamma}{2 \pi R^{2} J_{1}(k R)} J_{0}(k r), & r \leqslant R, \\ 0, & r \geqslant R,\end{cases}
$$

with $r$ the radial distance to the centre of the vortex, $R$ its radius, and $\Gamma$ its strength or circulation. $J_{0}$ and $J_{1}$ are Bessel functions of the first kind and $k R \approx 2.4048$ is the first non-zero root of $J_{0}$. The maximum of the vorticity is located at the centre of the monopole, where $J_{0}$ equals unity. (The vortex given by (23) is an exact, stationary solution of the inviscid vorticity equation (12) in an infinite domain, which satisfies the linear relationship $\omega=k^{2} \psi$ ). Viscous effects spread the vorticity over a larger area, so that the radius increases and the vorticity amplitude decreases as time evolves. The question addressed here is how this affects the motion of three monopoles in the initial configuration given by (8), and whether or not a collapse takes place between them. 
At the boundaries of the domain a so-called free-slip condition is used: the velocity perpendicular to the wall equals zero, whereas there is no restriction on the velocity parallel to the wall. In the case of a no-slip condition (i.e. the velocity equals zero everywhere at the wall) viscously generated vorticity at the walls will in general drastically affect the vortices. Using free-slip walls of course also affects the motion of the monopoles but to a lesser extent than no-slip walls (see van Geffen et $a .^{24}$, where the effect of walls on the evolution of a single monopole is studied).

The necessity of boundaries in the finite-difference calculations implies that the results cannot be quantitatively compared with results of the point vortex and contour-dynamics method of the previous sections; such a comparison can only be qualitative. It is, however, possible to compute the effect of free-slip walls on the motion of point vortices in a bounded domain by including the effects of (the infinite number of) mirror-images of these point vortices in the walls when solving (1) (e.g. Villat ${ }^{25}$, Müller $^{26}$ and Saffman ${ }^{27}$ ). The domain chosen for the computations presented below is $22 \times 22$ and centred around $(-1,1)$, which is roughly the centre of the trajectories the vortices follow. Figure 1 shows the trajectories of point vortices in this finite domain (short-dashed lines) for $t=0$ until $t=25$; the trajectories of point vortices in an infinite domain, given by (9), are shown in Figure 1 by solid lines. It is clear from this graph that the presence of free-slip walls slows down the point vortices with respect to point vortices in an infinite domain and their trajectories deviate already from the very beginning of the evolution. As the vortices come closer together, the velocities increase and they "overshoot": a collapse does not occur. Note that the strong negative point vortex (number 3 ) performs a small loop shortly before $t=25$; this can be seen more clearly in Fig. 9.

The larger the domain is, the better the trajectories of the point vortices in the finite domain coincide with those in an infinite domain, and the closer the angle at vortex 1 remains to 90 degrees. For instance, the distance between the corresponding point vortices at $t=25$ in a $77 \times 77$ domain and in an infinite domain is about 0.03 , and the angle at vortex 1 in the $77 \times 77$ domain is at that moment 90.94 degrees. Due to computer limitations, such large domains are unfortunately not possible for the finite-difference calculations. 
In the following the results of the finite-difference simulations are compared with the trajectories of the point vortices in the finite domain of $22 \times 22$, centred around $(-1,1)$, i.e. the short-dashed lines in Fig. 1 . The grid used has $512 \times 512$ cells and the time step $\Delta t$ is sufficiently small to ensure a stable computation.

\section{Results}

Consider first three Bessel monopoles of initial radius $R=0.25$ with initial strengths and positions given by (8) with $\alpha=\kappa=1$. Figure 7 shows the trajectories of the extrema of vorticity of these monopoles for $R e=1000$, compared with those of point vortices in the same bounded domain, and Fig. 8 shows the corresponding positions and shapes of the monopoles at six stages in the evolution. The trajectories were obtained by putting at $t=0$ passive tracers at the centres of the vortices (where the vorticity extrema are located) and following them in time during the calculation. Apparently, the passive tracers stay at the vorticity extrema during the motion: if this were not the case their paths would show several small loops. What is actually shown in Fig. 8 are the vorticity levels of +1 and -1 . These values are used here to define the "edges" of the monopoles, since due to viscous effects the monopoles after $t=0$ do not possess a clear sharp edge anymore. Viscosity causes a size increase of the monopoles as time evolves. Until about $t=20$ the three monopoles are still roughly circular and their trajectories are in good agreement with those of the corresponding point vortices in a bounded domain. At $t=20$ the distances between the monopoles has become of the order of their sizes and from hereon the monopoles are observed to deform ( $c f$. Fig. 3). After $t=20$ this deformation becomes more pronounced, especially for the initially weakest negative monopole (lightest shade of grey in Fig. 8). As a result, the drift velocities of the monopoles are smaller than those of the point vortices at the same moment, and the monopoles' paths deviate more and more from the trajectories of the corresponding point vortices. Clearly, a collapse between the monopoles into one single vortex does not take place.

After $t=25$ the two negative monopoles are observed to merge. The resulting two monopoles form an asymmetric dipolar vortex structure (with a strong negative and weaker 
positive part), which moves along a curved path. The monopoles gradually increase in size and their vorticity extrema decrease, both due to viscous effects, and hence they move with decreasing drift speed.

Both the viscosity and the initial size of the monopoles are important for the time scale of their evolution. The larger the viscosity is, the larger are the monopoles at a given time, and the earlier in the evolution the deformations are noticeable. The evolution of Bessel monopoles with initial radius $R=0.25$ has also been calculated for $R e=5000$ and $R e=10^{6}$ and the last part of their trajectories are shown in Fig. 9. For $R e=10^{6}$ the monopoles are almost circular and their trajectories coincide with those of the point vortices until close to $t=25$. The reason for this is of course that the size of the monopoles remains relatively small, as time evolves, so that the moment when the distance between them is of the order of their size is much later in the evolution than with $R e=1000$. Even for $R e=5000$ the distributed monopoles behave similar to the point vortices for a long time, considerably better than with $R e=1000$ ( $c f$. Fig. 7).

A computation with the finite-difference method without viscous effects, hence of eq. (12), reveals trajectories of the monopoles that are indistinguishable from those with $R e=10^{6}$. That even an inviscid computation does not lead exactly to the trajectories of the point vortices is caused by the finite size of the monopoles: at a certain moment the distance between the monopoles is of the order of their size, and from that moment on the shapes of these monopoles deform from their initially circular shape, and hence their trajectories deviate.

\section{CONCLUSIONS}

In this paper we have presented numerical simulations that demonstrate the influence of finite vortex sizes and viscosity effects on vortex interactions in a situation which leads to a vortex collapse in the point vortex case. Both in an infinite and in a finite domain, the results show a good agreement in the behaviour of finite size vortices with point vortices if their mutual distance is "sufficiently large". When these distances become of the order of the vortex sizes, their trajectories are seen to differ from those of the corresponding point 
vortices and the vortices start to deform. In the three-vortex configuration considered here, one vortex in particular deforms very dramatically, due to the growing internal and external strain. It is believed that this deformation into a long filament, which is gradually wrapped around the remaining vortices, prevents a collapse into one single final vortex.

The bigger the vortices initially are, the earlier they start to deform. In fact, only the time scale of the deformations is influenced by the size of the vortices; the deformations themselves are very similar for different initial sizes of the vortices. Viscosity causes the vortex sizes to grow as time evolves, thus resulting in earlier deformations than in the inviscid case.

Acknowledgement: One of us (JHGMvG) gratefully acknowledges financial support from the Non-Linear Systems programme of the Netherlands Organization for Scientific Research (NWO). This paper was partly written while VVM was visiting the Fluid Dynamics Laboratory in Eindhoven; this visit was also supported by NWO. We are also very grateful to D.G. Dritschel for his suggestions about the elliptical vortex patch model and for providing his numerical code of this model.

\section{References}

${ }^{1}$ W. Gröbli, "Specielle Probleme über die Bewegung geradliniger paralleler Wirbelfäden," Vierteljahr. Naturfor. Geselsch. Zürich, 22, 37, 129 (1877).

${ }^{2}$ H. Aref, N. Rott, and M. Thomann, "Gröbli's solution of the three-vortex problem," Ann. Rev. Fluid Mech., 24, 1 (1992).

${ }^{3}$ E.A. Novikov and Yu.B. Sedov, "Vortex collapse," Sov. Phys. JETP, 50 (2), 297 (1979).

${ }^{4}$ H. Aref, "Motion of three vortices," Phys. Fluids, 22, 393 (1979).

${ }^{5}$ Y. Kimura, "Similarity solution of two-dimensional point vortices," J. Phys. Soc. Japan, 56, 2024 (1987). 
${ }^{6}$ Y. Kimura, "Chaos and collapse of a system of point vortices," Fluid Dyn. Res., 3, 98 (1988).

${ }^{7}$ V.V. Meleshko and G.J.F. van Heijst, "Interacting two-dimensional vortex structures: point vortices, contour kinematics and stirring properties," Chaos, Solitons and Fractals, 4, 977 (1994).

${ }^{8}$ O.U. Velasco Fuentes, G.J.F. van Heijst, and B.E. Cremers, "Chaotic transport by dipolar vortices on a $\beta$-plane," J. Fluid Mech., 291, 139 (1995).

${ }^{9}$ O.U. Velasco Fuentes, G.J.F. van Heijst, and N.P.M. van Lipzig, "Unsteady behaviour of a topography-modulated tripole," J. Fluid Mech., 307, 11 (1996).

${ }^{10}$ G.K. Batchelor, "Introduction to Fluid Dynamics," Cambridge University Press, Cambridge (1967).

${ }^{11}$ D.G. Dritschel, "Contour surgery: A topological reconnection scheme for extended integrations using contour dynamics," J. Comput. Phys., 77, 240 (1988).

${ }^{12}$ D.G. Dritschel, "Contour dynamics and contour surgery: Numerical algorithms for extended, high-resolution modelling of vortex dynamics in two-dimensional, inviscid, incompressible flows," Comput. Phys. Rep., 10, 77 (1989).

${ }^{13}$ N.J. Zabusky, M.H. Hughes, and K.V. Roberts, "Contour dynamics for the Euler equations in two dimensions," J. Comput. Phys., 30, 96 (1979).

${ }^{14}$ P.W.C. Vosbeek and R.M.M. Mattheij, "Contour dynamics and symplectic time integration," J. Comput. Phys., 133 (2), 222 (1997).

${ }^{15}$ J.M. Sanz-Serna and M.P. Calvo, "Numerical Hamiltonian Problems," (Chapman \& Hall, London, 1994).

${ }^{16} \mathrm{~J}$. Weiss, "The dynamics of enstrophy transfer in two-dimensional hydrodynamics," Physica D, 48, 273 (1991). 
${ }^{17}$ J.C. McWilliams, "The emergence of isolated coherent vortices in turbulent flow," J. Fluid Mech., 146, 21 (1984).

${ }^{18}$ H. Lamb, "Hydrodynamics," 6th ed. (Cambridge University Press, Cambridge, 1932).

19 A.E.H. Love, "On the stability of certain vortex motions," Proc. Lond. Math. Soc., 35, 18 (1893)

${ }^{20}$ D.G. Dritschel, "The nonlinear evolution of rotating configurations of uniform vorticity," J. Fluid. Mech.,172, 157 (1986)

${ }^{21}$ D.G. Dritschel and M. de la Torre Juárez, "The instability and breakdown of tall columnar vortices in a quasi-geostrophic fluid," J. Fluid. Mech., 328, 129 (1996)

${ }^{22}$ P. Orlandi, "Vortex dipole rebound from a wall," Phys. Fluids A, 2, 1429 (1990).

${ }^{23}$ R. Verzicco, J.B. Flór, G.J.F. van Heijst, and P. Orlandi, "Numerical and experimental study of the interaction between a vortex dipole and a circular cylinder," Exp. Fluids, 18, 153 (1995).

${ }^{24}$ J.H.G.M. van Geffen, V.V. Meleshko, and G.J.F. van Heijst, "Motion of a two-dimensional monopolar vortex in a bounded rectangular domain," Phys. Fluids, 8, 2393 (1996).

${ }^{25}$ H. Villat, "Leçons sur la Théorie des Tourbillons," Gauthier-Villars, Paris (1930).

${ }^{26}$ W. Müller, "Bewegung von Wirbeln in einer idealen Flüssigkeit inter dem Einfluss von ebenen Wänden," Z. angew. Math. Mech, 10, 227 (1930).

${ }^{27}$ P.G. Saffman, "Vortex Dynamics," Cambridge University Press, Cambridge (1992). 


\section{Figures}

Figure 1 The trajectories of the point vortices in an infinite domain (solid lines) and in a bounded domain of size $22 \times 22$ with free slip walls (short-dashed lines) with initial positions (indicated by the numbers 1,2 and 3) and strengths according to (8). The initial locations of the point vortices are connected with long-dashed lines to show the triangle formed by the three vortices. Symbols are placed at the positions of the vortices at time $t=0,5,10,15,20$ and 25 (and $t=25.395$ in the infinite domain case).

Figure 2 The trajectories of the centres of three initially circular patches of uniform vorticity, with radius $R=0.50$ and initial locations and strengths according to (8) (solid lines). The trajectories were determined by computing for each patch at each time step the centre of vorticity using a contour integral representation. Also drawn are the trajectories of the corresponding point vortices (dashed lines). Symbols are again placed at the positions of the vortices at time $t=0,5,10,15,20$ and 25 .

Figure 3 Positions and shapes (solid lines) of the monopoles of Fig. 2 at six moments in time from $t=20 \mathrm{on}$. The grey shading represents the spatial distribution of the strain rate; dark regions have strong strain rate.

Figure 4 The value of $Q$ plotted for two Kirchhoff vortices with different aspect ratio, both with vorticity $\omega=1$. In (a) the aspect ratio of the vortex is equal to 2 , in (b) it is equal to 4 .

Figure 5 Six stages in the flow evolution of the three monopoles modelled by the elliptical vortex patch model. The initial configuration is exactly the same as in Fig. 3. The edges of the elliptical vortices are indicated with solid lines. The cross in the centre of the vortices displays both the magnitude and the orientation of the external strain with extension along the solid axis and compression along the dashed axis.

Figure 6 A part of the trajectories of the monopoles with uniform vorticity with initial radius $R=0.25$ (a) and $R=0.75$ (b) (solid lines) compared with the trajectories 
of the corresponding point vortices (dashed lines). The numbers 1,2 and 3 indicate the trajectories of the corresponding vortices. Symbols are placed at the positions of the vortices at time $t=0,5,10,15,20$ and 25 . Note the difference in domain size of the two pictures.

Figure $7 \quad$ Trajectories of the centres of three Bessel monopoles initially of radius $R=0.25$ and with initial positions and strengths given by (8) (solid lines) and of the corresponding point vortices (dashed lines) in the same bounded domain. Symbols are placed at the positions of the vortices at $t=0,5,10,15,20$ and 25 .

Figure 8 Position and shape of the monopoles of Fig. 7 at six stages in the flow evolution for $R e=1000$. The shapes of the monopoles are defined by the vorticity levels +1 and -1 ; for clarity other vorticity levels are not shown. The darkest vortex is the strong negative monopole, the lightest is the weak negative monopole. The part of the domain shown here is the same as that in Fig. 7 .

Figure 9 Part of the trajectories of Bessel monopoles with initial radius $R=0.25$ for $R e=5000$ (a) and $R e=10^{6}$ (b) (solid lines) compared with the trajectories of the corresponding point vortices (dashed lines). In both cases vortex 1 enters the shown part of the domain at the top at about $t=20$, vortex 2 enters at the right at about $t=22$, and for vortex 3 the full trajectory is shown. Symbols are placed at the positions of the vortices at $t=0,5,10,15,20$ and 25 . 


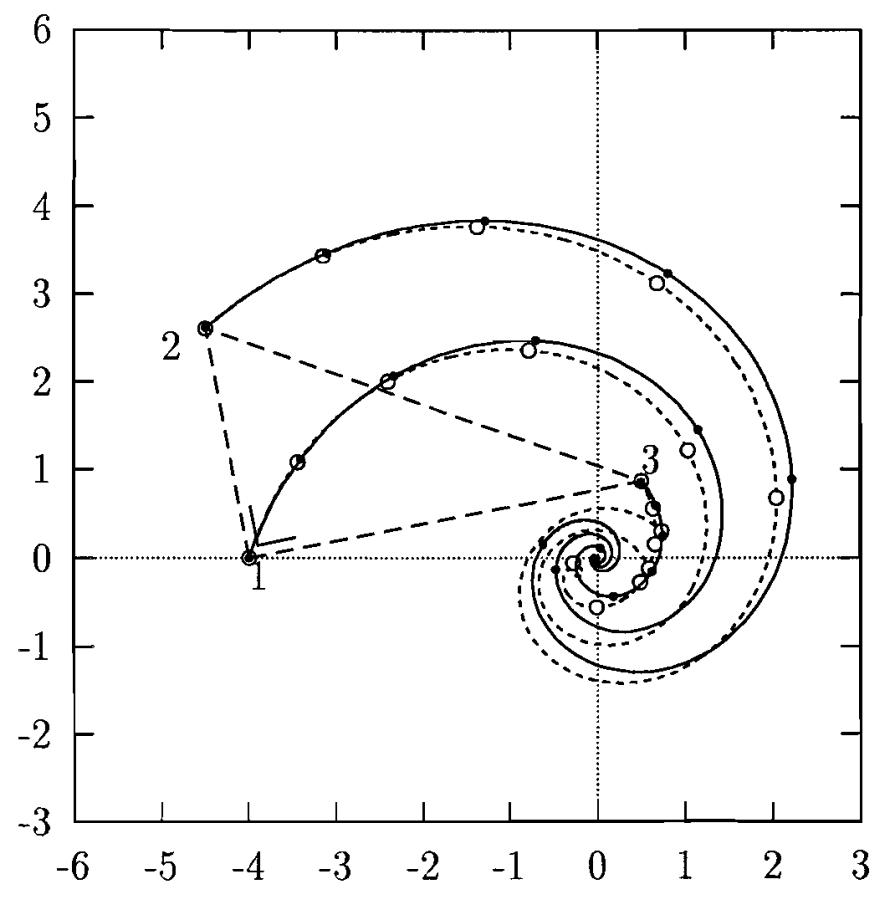

Figure 1 P.W.C. Vosbeek, J.H.G.M. van Geffen, V.V. Meleshko, G.J.F. van Heijst 


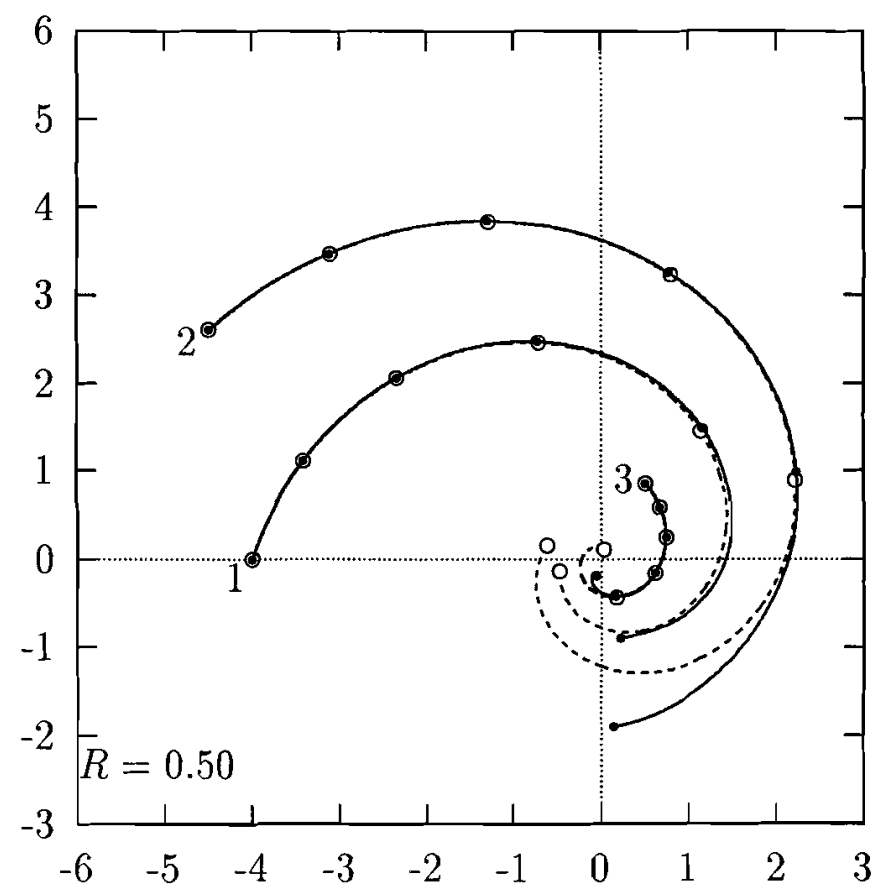

Figure 2 P.W.C. Vosbeek, J.H.G.M. van Geffen, V.V. Meleshko, G.J.F. van Heijst 

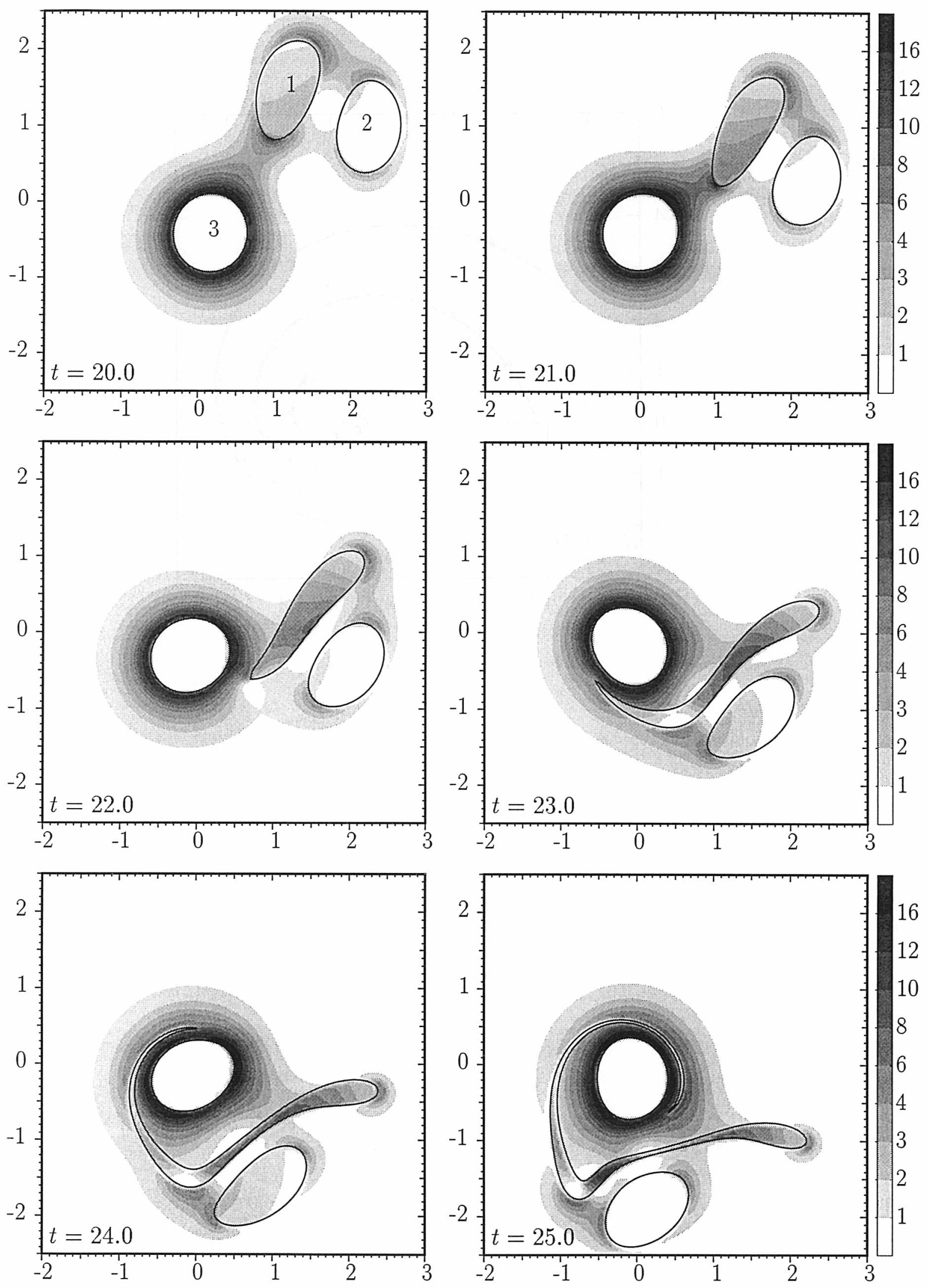

Figure 3 P.W.C. Vosbeek, J.H.G.M. van Geffen, V.V. Meleshko, G.J.F. van Heijst 


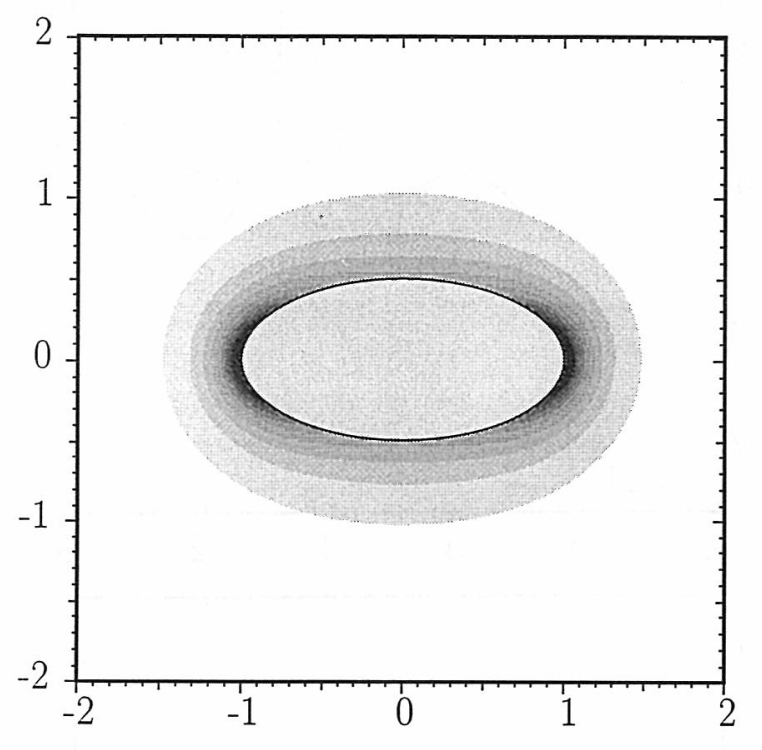

(a)

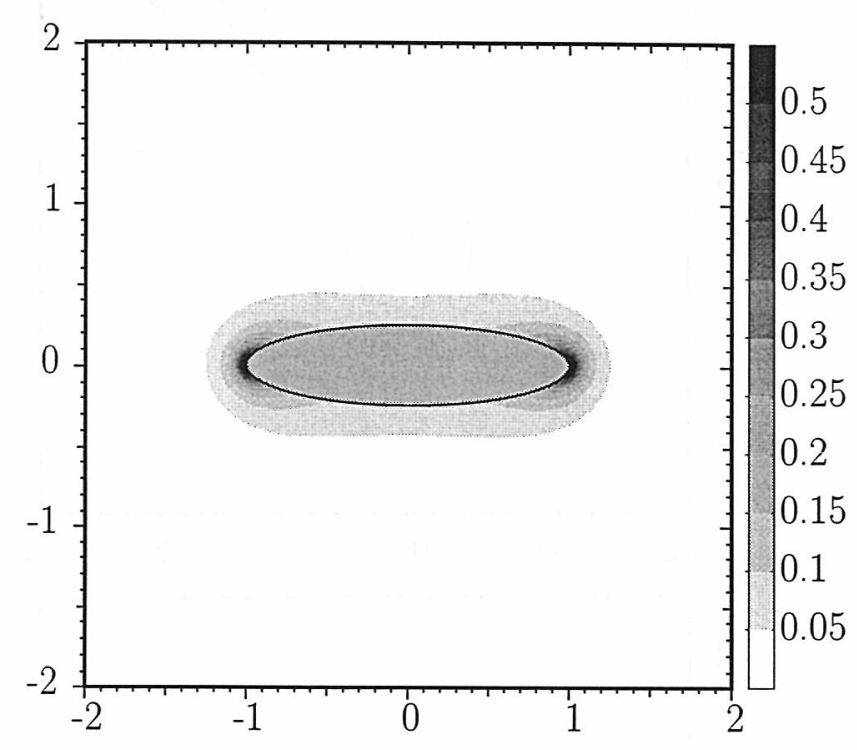

(b)

Figure 4 P.W.C. Vosbeek, J.H.G.M. van Geffen, V.V. Meleshko, G.J.F. van Heijst 

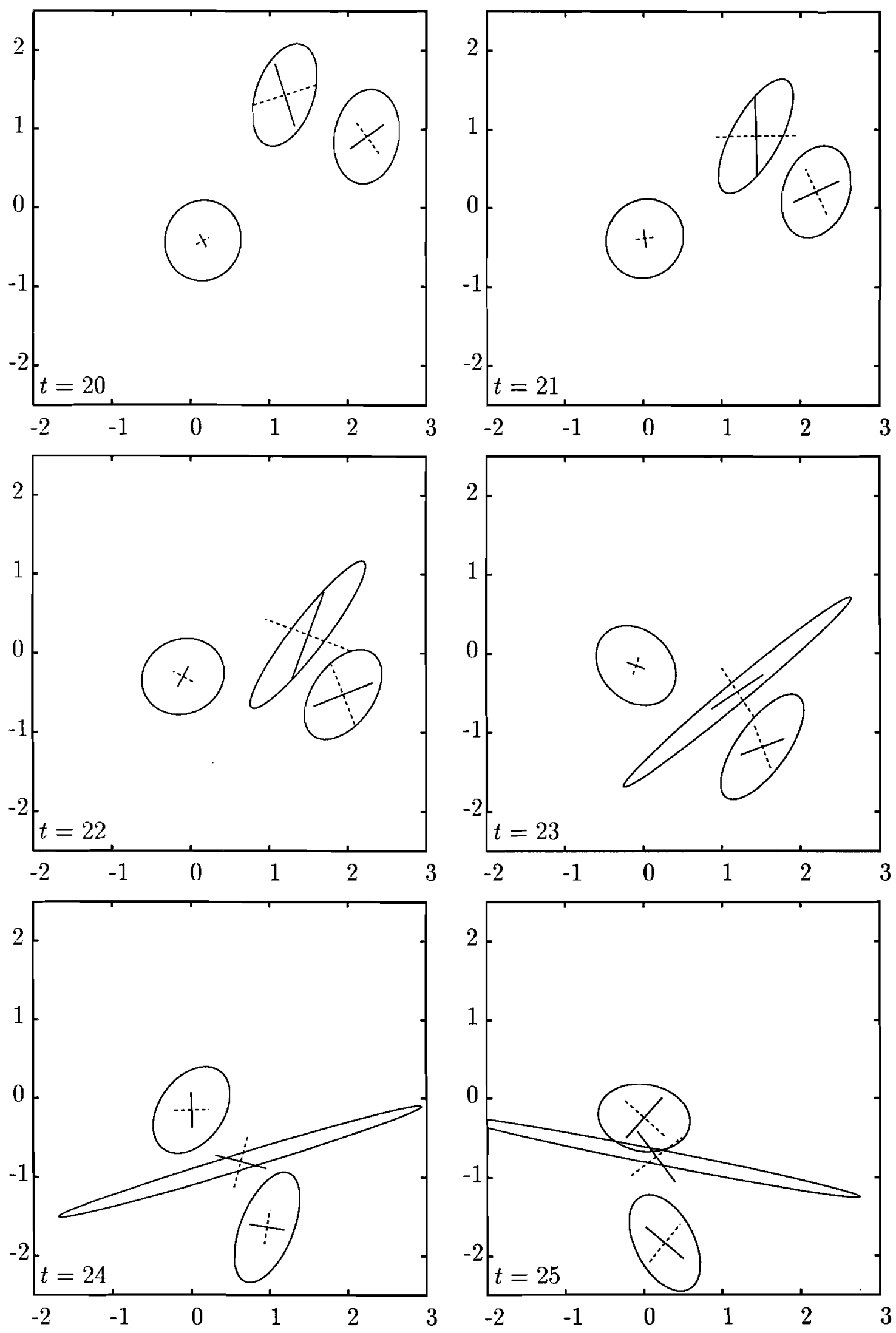

Figure 5 P.W.C. Vosbeek, J.H.G.M. van Geffen, V.V. Meleshko, G.J.F. van Heijst 


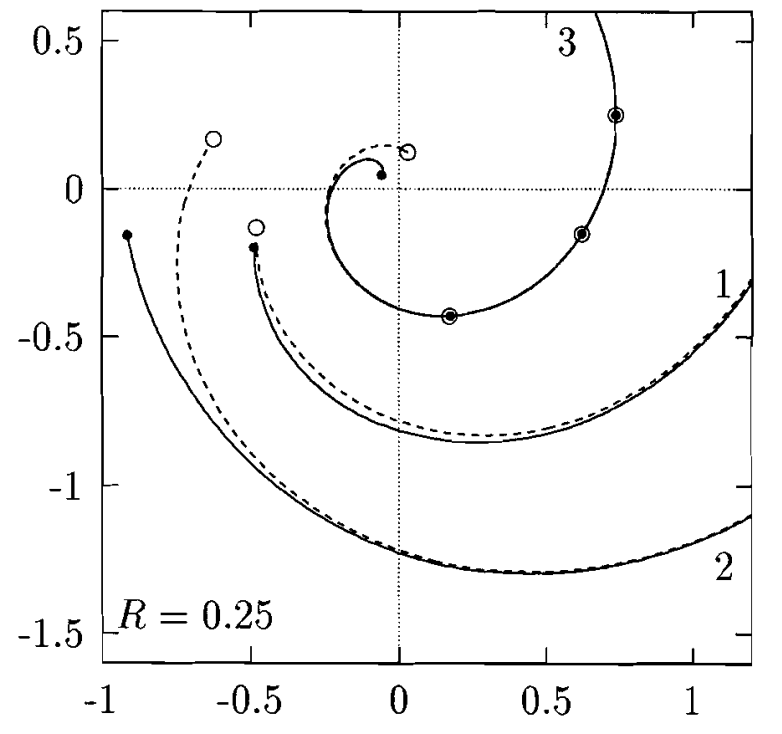

(a)

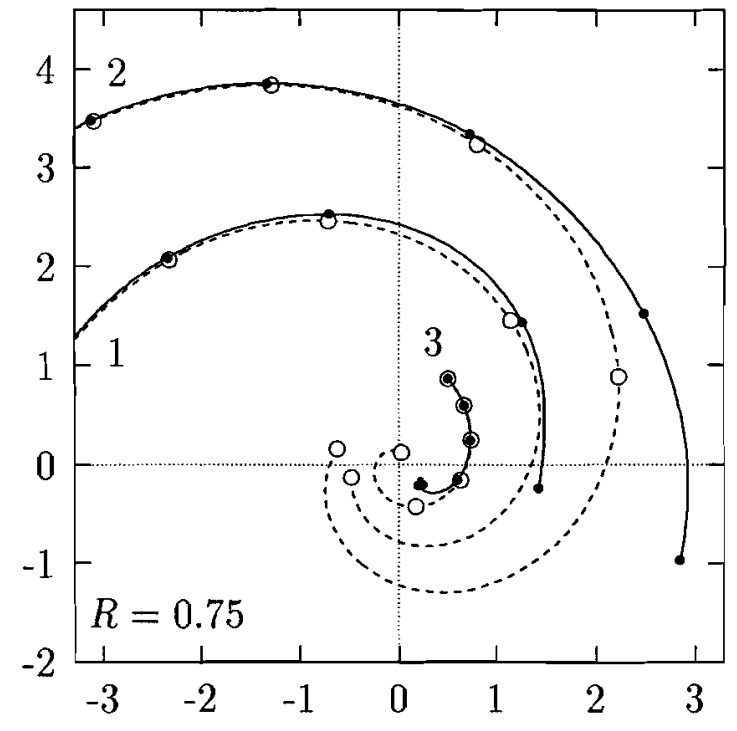

(b)

Figure 6 P.W.C. Vosbeek, J.H.G.M. van Geffen, V.V. Meleshko, G.J.F. van Heijst 


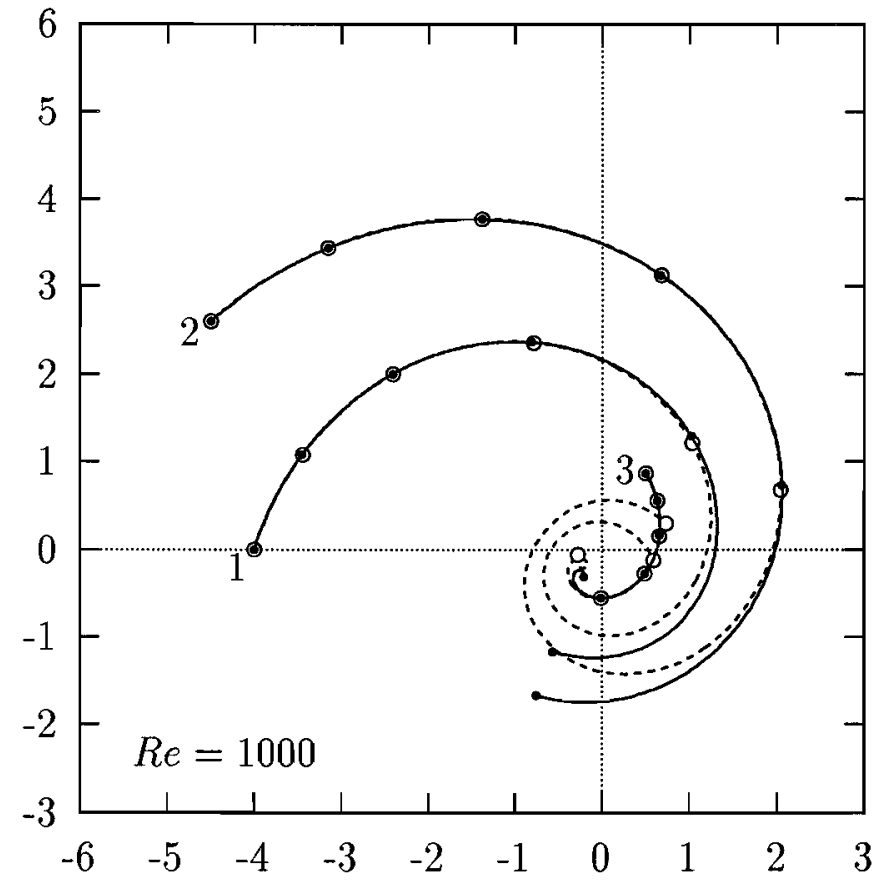

Figure 7 P.W.C. Vosbeek, J.H.G.M. van Geffen, V.V. Meleshko, G.J.F. van Heijst 

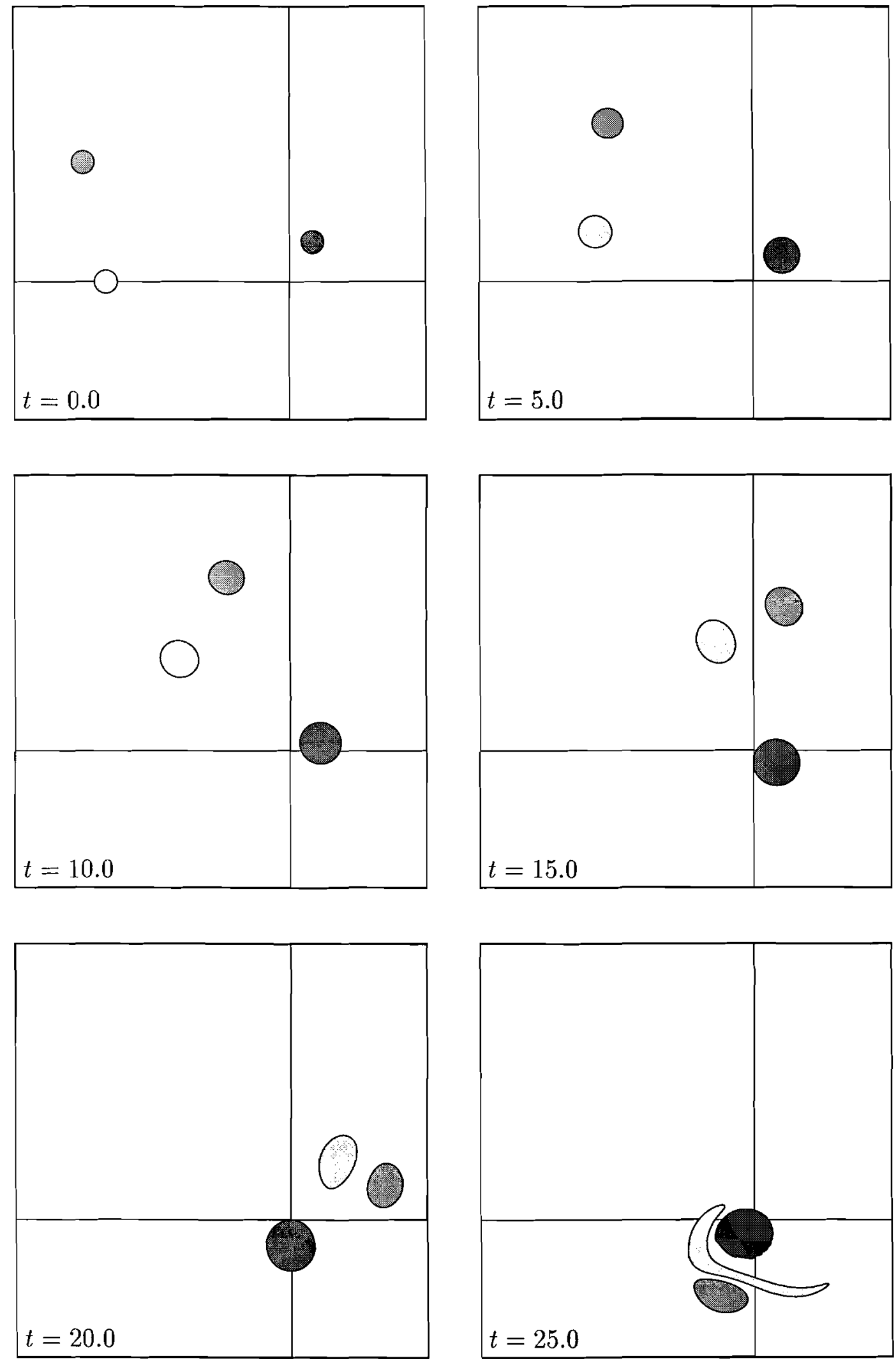

Figure 8 P.W.C. Vosbeek, J.H.G.M. van Geffen, V.V. Meleshko, G.J.F. van Heijst 


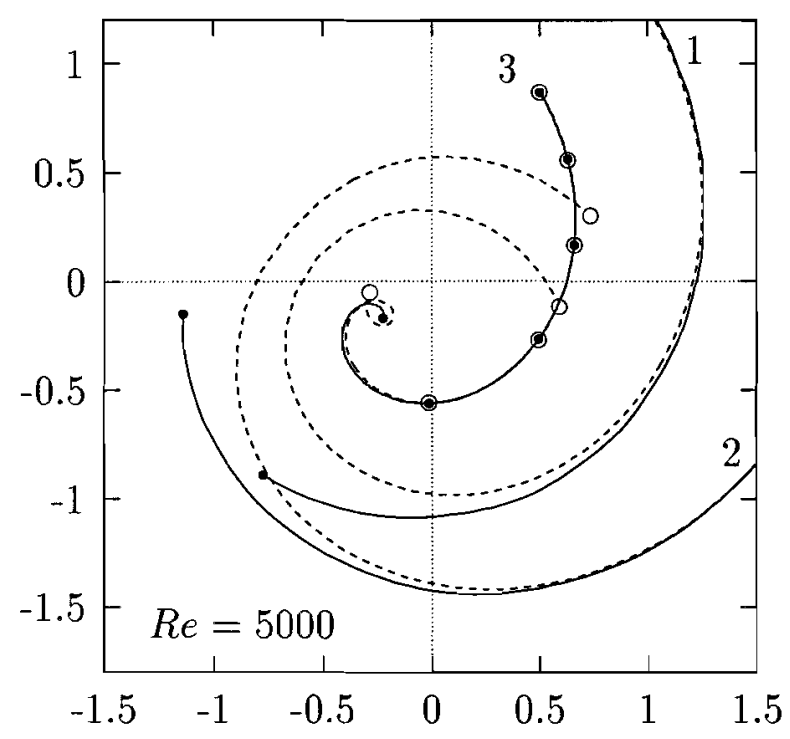

(a)

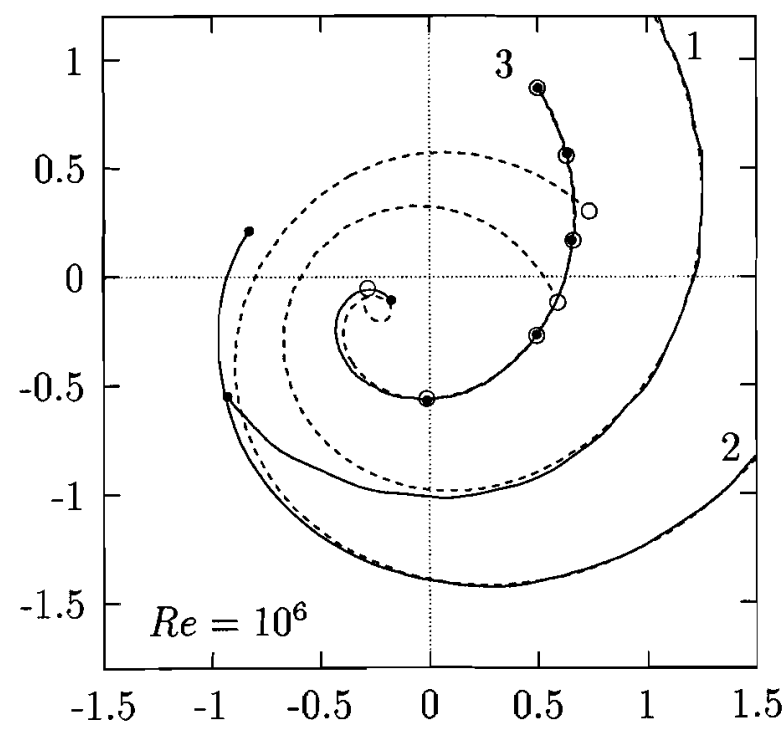

(b)

Figure 9 P.W.C. Vosbeek, J.H.G.M. van Geffen, V.V. Meleshko, G.J.F. van Heijst 


\section{PREVIOUS PUBLICATIONS IN THIS SERIES:}

\begin{tabular}{|c|c|c|c|}
\hline Number & Author(s) & Title & Month \\
\hline $96-26$ & J. de Graaf & $\begin{array}{l}\text { Spaces of harmonic functions and } \\
\text { evolution equations in them }\end{array}$ & $\overline{\text { December }{ }^{\circ} 96}$ \\
\hline $96-2 i$ & $\begin{array}{l}\text { R.M.M. Mattheij } \\
\text { S.J. Wright }\end{array}$ & $\begin{array}{l}\text { Parallel algorithms for parameter } \\
\text { identification in odes }\end{array}$ & December 96 \\
\hline $9--01$ & $\begin{array}{l}\text { J.H.P.A. Martens } \\
\text { J.C. Reijenga } \\
\text { J.H.M. ten Thije } \\
\text { Boonkkamp } \\
\text { R.M.M. Mattheij } \\
\text { F.M. Everaerts }\end{array}$ & $\begin{array}{l}\text { Transient modelling of capillary } \\
\text { electrophoresis. Isotachophoresis }\end{array}$ & January 97 \\
\hline $97-02$ & A.A. Reusken & $\begin{array}{l}\text { Approximate cyclic reduction } \\
\text { preconditioning }\end{array}$ & February ' 97 \\
\hline $9 \tau-0.3$ & J.J.A.M. Brands & $\begin{array}{l}\text { Asymptotics of non-Laplacian } \\
\text { integrals }\end{array}$ & February ' 97 \\
\hline $9 \tau-04$ & $\begin{array}{l}\text { M.J. Noot A.C. Telea } \\
\text { J.K.M. Jansen } \\
\text { R.M.M. Mattheij }\end{array}$ & $\begin{array}{l}\text { Real Time Numerical Simulation } \\
\text { and Visualization of Electrochemical } \\
\text { Drilling }\end{array}$ & March '97 \\
\hline $9 \bar{\imath}-0 j$ & H.J.C. Huijberts & $\begin{array}{l}\text { Characterization of static feedback } \\
\text { realizable transfer functions for non- } \\
\text { linear control systems }\end{array}$ & May ' 97 \\
\hline $97-06$ & B. van $t$ Hof & $\begin{array}{l}\text { Numerical Simulation of Unsteady } \\
\text { Premixed Laminar Flames }\end{array}$ & July '9 9 \\
\hline $9 \tau-0 T$ & $\begin{array}{l}\text { P.W.C. Vosbeek } \\
\text { J.H.G.M. van Geffen } \\
\text { V.V. Meleshko } \\
\text { G.J.F. van Heijst }\end{array}$ & $\begin{array}{l}\text { Collapse interactions of finite-sized } \\
\text { two-dimensional vortices }\end{array}$ & July '97 \\
\hline
\end{tabular}

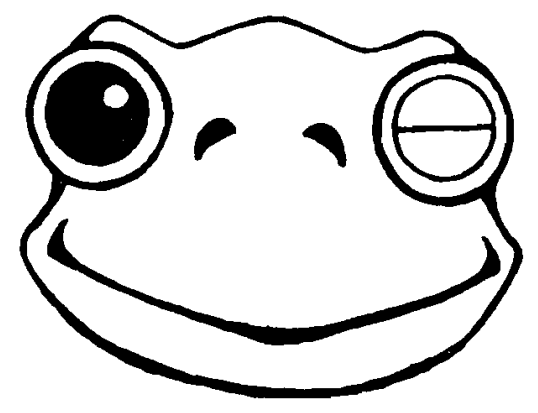

